\title{
Human phospholamban null results in lethal dilated cardiomyopathy revealing a critical difference between mouse and human
}

\author{
Kobra Haghighi, ${ }^{1}$ Fotis Kolokathis, ${ }^{2}$ Luke Pater, ${ }^{1}$ Roy A. Lynch, ${ }^{3}$ Michio Asahi, ${ }^{4}$ \\ Anthony O. Gramolini, ${ }^{4}$ Guo-Chang Fan, ${ }^{1}$ Dimitris Tsiapras, ${ }^{2}$ Harvey S. Hahn, ${ }^{3}$ \\ Stamatis Adamopoulos, ${ }^{2}$ Stephen B. Liggett, ${ }^{3}$ Gerald W. Dorn II, ${ }^{3}$ David H. MacLennan, ${ }^{4}$ \\ Dimitrios T. Kremastinos, ${ }^{2}$ and Evangelia G. Kranias ${ }^{1}$

\footnotetext{
${ }^{2}$ Onassis Cardiac Surgery Center, Athens, Greece

${ }^{3}$ Department of Medicine, Cardiology and Pulmonary Divisions, University of Cincinnati, College of Medicine, Cincinnati, Ohio, USA

${ }^{4}$ Banting and Best Department of Medical Research, University of Toronto, Toronto, Ontario, Canada
} \\ ${ }^{1}$ Department of Pharmacology and Cell Biophysics, University of Cincinnati, College of Medicine, Cincinnati, Ohio, USA
}

\begin{abstract}
In human disease and experimental animal models, depressed $\mathrm{Ca}^{2+}$ handling in failing cardiomyocytes is widely attributed to impaired sarcoplasmic reticulum (SR) function. In mice, disruption of the PLN gene encoding phospholamban (PLN) or expression of dominant-negative PLN mutants enhances SR and cardiac function, but effects of PLN mutations in humans are unknown. Here, a T116G point mutation, substituting a termination codon for Leu-39 (L39stop), was identified in two families with hereditary heart failure. The heterozygous individuals exhibited hypertrophy without diminished contractile performance. Strikingly, both individuals homozygous for L39stop developed dilated cardiomyopathy and heart failure, requiring cardiac transplantation at ages 16 and 27. An over 50\% reduction in PLN mRNA and no detectable PLN protein were noted in one explanted heart. The expression of recombinant PLN-L39stop in human embryonic kidney (HEK) 293 cells and adult rat cardiomyocytes showed no PLN inhibition of SR $\mathrm{Ca}^{2+}$-ATPase and the virtual absence of stable PLN expression; where PLN was expressed, it was misrouted to the cytosol or plasma membrane. These findings describe a naturallyoccurring loss-of-function human PLN mutation (PLN null). In contrast to reported benefits of PLN ablation in mouse heart failure, humans lacking PLN develop lethal dilated cardiomyopathy.

This article was published online in advance of the print edition. The date of publication is available from the JCI website, http://www.jci.org. J. Clin. Invest. 111:869-876 (2003). doi:10.1172/JCI200317892.
\end{abstract}

\section{Introduction}

Heart failure is a major cause of death and disability worldwide. In the United States, it is the only form of cardiovascular disease increasing in incidence and prevalence, with nearly 400,000 new cases annually (1). Although targeting the neurohormonal axis in heart failure with $\beta$-adrenergic receptor blockers and

Received for publication January 17, 2003, and accepted in revised form January 28, 2003.

Address correspondence to: Evangelia G. Kranias, Department of Pharmacology and Cell Biophysics, University of Cincinnati, College of Medicine, 231 Albert Sabin Way, Cincinnati, Ohio 45267-0575, USA. Phone: (513) 558-2377;

Fax: (513) 558-2269; E-mail: litsa.kranias@uc.edu.

Conflict of interest: The authors have declared that no conflict of interest exists.

Nonstandard abbreviations used: sarcoplasmic reticulum (SR); phospholamban (PLN); human embryonic kidney (HEK); cardiac SR Ca ${ }^{2+}$ ATPase (SERCA2a); interventricular septal thickness (IVS); anterior wall thickness (AW); posterior wall thickness (PW); left ventricular end systolic diameter (LVESD); left ventricular end diastolic diameter (LVEDD); left ventricular mass (LVM); wild-type PLN (PLN-WT); shortening/contraction $(+\mathrm{dL} / \mathrm{dt})$; relengthening/relaxation $(-\mathrm{dL} / \mathrm{dt}) ; \beta_{1}$-adrenergic receptor ( $\left.\beta \mathrm{AR}\right)$. angiotensin-converting-enzyme inhibitors has improved its overall prognosis, heart failure continues to have an overall 5-year mortality rate of approximately $50 \%$ (2). Hence, novel therapeutic targets are needed in this syndrome.

Altered cardiomyocyte $\mathrm{Ca}^{2+}$ cycling is widely recognized as contributing to impaired contractile performance in human and experimental heart failure, including idiopathic dilated cardiomyopathy $(3,4)$. Coordinated regulation of cytosolic $\mathrm{Ca}^{2+}$ by the sarcoplasmic reticulum (SR) of myocytes is required during each cycle of cardiac contraction and relaxation. Cytosolic $\mathrm{Ca}^{2+}$ is sequestered into the SR lumen by cardiac SR Ca ${ }^{2+}$-ATPase (SERCA2a), permitting muscle relaxation; subsequently, the stored $\mathrm{Ca}^{2+}$ is released through ryanodine receptor channels to activate myofilament contraction (5). The activity of SERCA2a is reversibly regulated by phospholamban (PLN), a 52-amino-acid phosphoprotein (6). Dephosphorylated PLN interacts with SERCA2a and inhibits $\mathrm{Ca}^{2+}$ pump activity, whereas protein kinase A phosphorylation of PLN through the $\beta$-adrenergic pathway relieves 
its inhibitory effects and augments relaxation (6). Experimental data obtained in human and animal heart failure have demonstrated associations between increased PLN inhibition of SR $\mathrm{Ca}^{2+}$ sequestration that is, impaired $\mathrm{Ca}^{2+}$ reuptake - and contractile dysfunction, suggesting a causal role for altered $\mathrm{Ca}^{2+}$ cycling in the development or progression of heart failure (7-9). Consistent with this notion, reversal of PLN inhibitory activity by in vivo cardiac adenoviral gene delivery improves cardiac function in the cardiomyopathic hamster (10), and mice deficient in PLN demonstrate enhanced $\mathrm{Ca}^{2+}$ reuptake, increased $\mathrm{Ca}^{2+}$ transient amplitude, and improved contractility (11-13). In the PLN-null mouse, supernormal cardiac function is a permanent feature that does not, over time, compromise exercise endurance or abbreviate life span $(13,14)$. In human hearts, however, chronic cardiac hypercontractily has been implicated as a cause of dilated cardiomyopathy $(15,16)$.

Since a genetic basis for "idiopathic" dilated cardiomyopathy has been established by pedigree studies showing that up to $30 \%$ of cases have a heritable basis (17), we sought herein to identify naturally occurring mutation(s) in the human PLN gene that could perturb cardiac $\mathrm{Ca}^{2+}$ cycling and, therefore, contribute to dilated cardiomyopathy. A truncation mutation was identified that resulted in dramatically diminished myocardial PLN protein content and consequent loss of PLN inhibition of SERCA2a, and in homozygous individuals was associated with development of heart failure and early mortality. These findings demonstrate that in contrast to mice in which phospholamban deficiency enhances myocardial inotropy and lusitropy without adverse effects, PLN is essential for cardiac health in humans, and its absence results in lethal heart failure.

\section{Methods}

Mutation identification and Tru9I restriction endonuclease screening. The protocol was approved by the institutional review board of the Onassis Cardiac Surgery Center or the University of Cincinnati College of Medicine, and the subjects provided written informed consent. Genomic DNA was isolated either from whole blood or from paraffin blocks containing heart tissue. The PLN gene consists of two exons, and the coding region is totally contained in exon 2 (18). A 348-bp fragment containing the entire PLN coding region in exon 2 was amplified by PCR, using 60 ng of genomic DNA and a high-fidelity Taq polymerase. The primers were sense $5^{\prime}$-TCTCATATTTGGCTGCC- $3^{\prime}$ and antisense $5^{\prime}$-ATTGTTTTCCTGTCTGC- $3^{\prime}$ tagged with M13 forward and reverse primer sequences, respectively. The conditions were as follows: one cycle at $94^{\circ} \mathrm{C}$ for 3 minutes, linked to 30 cycles at $94^{\circ} \mathrm{C}$ for 1 minute, $46^{\circ} \mathrm{C}$ for 1 minute, and $72^{\circ} \mathrm{C}$ for 1 minute, followed by one cycle at $94^{\circ} \mathrm{C}$ for 1 minute, $53^{\circ} \mathrm{C}$ for 1 minute, and $72^{\circ} \mathrm{C}$ for 10 minutes. PLN DNA was sequenced using automated dye-primer chemistry.
The generated sequences were compared with the reported human PLN sequences by a computational method, and the electropherograms were inspected individually for confirmation. The mutation results in a loss of a Tru9I restriction endonuclease site. Thus, for rapid screening, the PCR products were digested with Tru9I, which provided four fragments of 147, 87, 61 , and $53 \mathrm{bp}$ from wild-type templates and three fragments of 234, 61, and $53 \mathrm{bp}$ from T116G homozygotes after $2 \%$ agarose gel electrophoresis. Nevertheless, the PLN DNA from all subjects in kindreds I and II was sequenced as well.

Echocardiography. Comprehensive 2D and Doppler echocardiography was performed (19). Left ventricular dimension (interventricular septal thickness [IVS], anterior wall thickness [AW], posterior wall thickness [PW], and left ventricular end systolic diameter [LVESD] and diastolic diameter [LVEDD]) was measured with M-mode echocardiography by using the leading edge-to-leading edge convention. Left ventricular mass (LVM) was determined by using the Troy formula: $\operatorname{LVM}(\mathrm{g})=1.05[(\mathrm{LVEDD}+\mathrm{IVS}+$ $\left.\mathrm{PW})^{3}-\mathrm{LVEDD}^{3}\right](19)$.

$\mathrm{Ca}^{2+}$ transport and immunofluorescence in HEK-293 cells. Mutagenesis of PLN cDNA was carried out as described previously $(20,21)$. Wild-type and mutant PLN cDNAs were ligated into the XbaI and SalI sites of the PMT2 expression vector for amplification, and plasmid DNA was purified using Qiagen columns. PLN and SERCA2a cDNA in PMT2 vectors was cotransfected (8 $\mu \mathrm{g}$ of each cDNA per dish, 1:1 ratio) into human embryonic kidney (HEK) 293 cells using the $\mathrm{Ca}^{2+}$ phosphate precipitation method. Cells were harvested 24 or 48 hours after transfection, and microsomes were prepared and assayed for $\mathrm{Ca}^{2+}$ transport activity or immunoblotting with the PLN monoclonal antibody ID11 (gift from Robert Johnson, Merck Research Laboratories, Whitehouse Station, New Jersey, USA) (21).

For immunofluorescence experiments, HEK-293 cells were grown on $18 \times 18 \mathrm{~mm}$ glass coverslips and transfected with $400 \mathrm{ng}$ of PLN cDNA per $35-\mathrm{mm}$ plate using Superfect (Qiagen, Mississauga, Ontario, Canada). At 24-48 hours after transfection, cells were fixed and processed for immunofluorescence using the PLN antibody ID11 and a fluorescein isothiocyanate-conjugated secondary antibody (Jackson ImmunoResearch Laboratories Inc., West Grove, Pennsylvania, USA). Images were collected using a Leica DM IRBE inverted microscope equipped with a Leica TCS SP laser scanning confocal system (Leica Microsystems Inc., Richmond Hill, Ontario, Canada).

PLN gene expression. Northern blots of total cardiac RNA $(20 \mu \mathrm{g})$ from the explanted heart of proband III-4 were probed with the radiolabeled 60-mer oligonucleotide, antisense to the human PLN coding region. The probed blots were stained with methylene blue to demonstrate RNA integrity and loading. 
Recombinant adenovirus. The recombinant adenovirus construction protocol was described previously (22). Briefly, the mutant L39stop cDNA was cloned into the shuttle vector pAdTrack-CMV (gift from Roger J. Hajjar, Harvard Medical School, Boston, Massachusetts, USA), linearized with restriction endonuclease PmeI, and cotransfected into E. coli BJ5183 cells (Stratagene, La Jolla, California, USA) with the adenoviral backbone plasmid pAdEasy-1 (gift from Bert Vogelstein, The Johns Hopkins Oncology Center, Baltimore, Maryland, USA). The recombinants were verified by restriction endonuclease mapping with PacI. The linearized recombinant plasmid was transfected into an adenovirus packaging cell line (HEK-293 cells) using Lipofectamine 2000 (Invitrogen, Carlsbad, California, USA). Recombinant adenovirus, denoted Ad.PLN-L39stop, which encoded for both PLN-L39stop mutant protein and GFP controlled by separate CMV promoters, was harvested after 7 days and then amplified and purified by $\mathrm{CsCl}$ gradient centrifugation.

Contractile mechanics of adult rat myocytes. Myocytes from adult Sprague-Dawley male rats (approximately $300 \mathrm{~g}$; Harlan Laboratory, Indianapolis, Indiana, USA) were isolated from left ventricular tissue (23). The cells were resuspended in Claycomb medium supplemented with $0.2 \%$ BSA, penicillin $(100 \mathrm{U} / \mathrm{ml})$, and streptomycin (100 $\mu \mathrm{g} / \mathrm{ml}$ ) at a density of $10^{5}$ cells per $100-\mathrm{cm}^{2}$ lamin-precoated dish or in $2 \times 10^{4}$ cells per well of 6 -well plates with lamin-precoated glass coverslips. After 2 hours, myocytes were infected with the adenoviruses Ad.PLNL39stop, Ad.PLN-WT, or Ad.GFP at a multiplicity of infection of 500. Twenty-four hours after infection, myocytes adherent to coverslips were incubated in 1 $\mathrm{mM} \mathrm{Ca}{ }^{2+}$-Krebs Henseleit buffer, and mechanical or $\mathrm{Ca}^{2+}$-kinetic parameters in fura-2AM-loaded $(2 \mu \mathrm{M})$ myocytes were determined. Fields of myocytes were chosen at random and stimulated at $0.5 \mathrm{~Hz}$. $\mathrm{Ca}^{2+}$ transients were calibrated as described (24). Data were analyzed by Felix computer software (Photon Technology International, Lawrenceville, New Jersey, USA).

Biochemical assays. Quantitative immunoblotting of cardiac homogenates was used to determine the levels of PLN and SR $\mathrm{Ca}^{2+}$-handling proteins, as described previously (25).

Histology. Left-ventricular samples were collected from patients during heart transplantation, fixed overnight in $10 \%$ formalin, buffered with PBS, dehydrated in $70 \%$ ethanol, and transferred to xylene and then into paraffin. Paraffin-embedded heart samples were sectioned at $4 \mu \mathrm{m}$ and stained with Masson's trichrome.

Statistics. Data are presented as means \pm SEM. Comparisons were made using Student's $t$ test as appropriate. A $P$ value of less than 0.06 was considered statistically significant.

\section{Results}

Clinical evaluation. Seventy-six patients with dilated cardiomyopathy and 30 normal subjects were recruited from the Onassis Cardiac Surgery Center. Three patients had undergone heart transplantation, and two were on the waiting list. The average ejection fraction, determined by echocardiography, was $22.9 \% \pm 9.91 \%$. Comorbid conditions in the cohort included hypertension $(8 \%)$, diabetes $(6 \%)$, hypercholesterolemia (12\%), and atrial fibrillation (12\%).

Identification of a mutant PLN gene in cardiomyopathy pedigrees. Sequencing of the PLN gene coding region from the 76 unrelated individuals with dilated cardiomyopathy revealed one patient homozygous for a T-to-G transversion at nucleic acid 116 (T116G) (Figure 1a). This mutation was not found in 30 unrelated normal subjects matched in age and ethnic background or in 220 unrelated normal subjects. The mutation converts the codon for Leu-39 to the premature stop codon TGA (denoted PLNL39stop), truncating the 52-amino-acid protein in transmembrane domain II, which is highly conserved among species (18) and involved in PLN regulation of SERCA2a affinity for $\mathrm{Ca}^{2+}(6)$. Using alaninescanning mutagenesis, we have previously shown
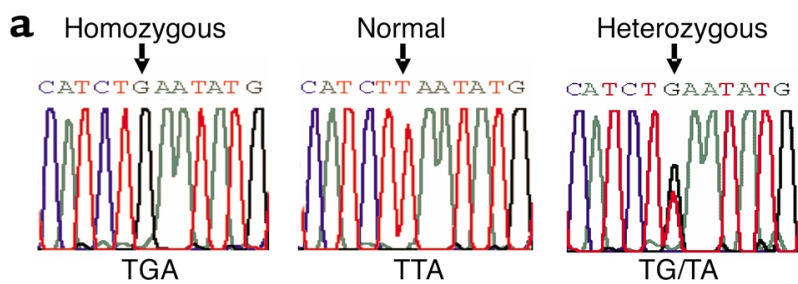

b

TGA

TTA

$\mathrm{TG} / \mathrm{TA}$

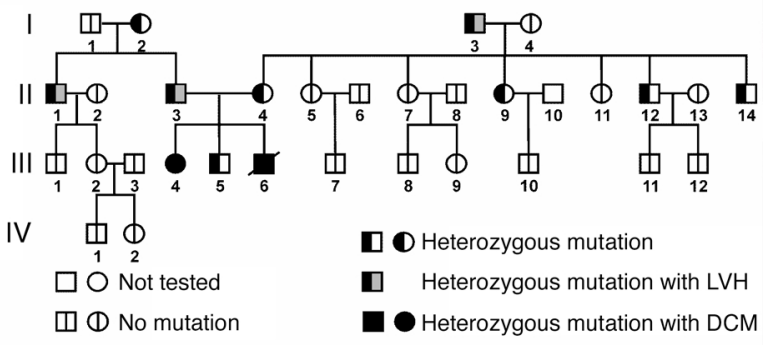

C

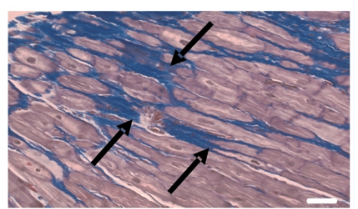

Proband III-4

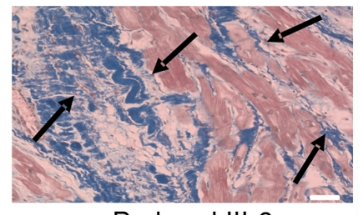

Proband III-6

\section{Figure 1}

Mutation in the PLN gene and analysis of inheritance in kindred I. (a) Partial nucleotide sequences of the PLN coding region in normal subjects and in patients with dilated cardiomyopathy who were homozygous or heterozygous for the T116G transversion, which converts the codon for Leu-39 (TTA) to a stop codon (TGA). (b) Pedigree for the presence or absence of the T116G mutation in kindred I. Probands III-4 and III-6, who were homozygous for the L39stop mutation, were diagnosed with dilated cardiomyopathy and required cardiac transplantation. Squares represent males and circles represent females. A line denotes that the patient is deceased. (c) Histological analysis of explanted hearts from probands III-4 and III-6 (who were homozygous for the L39stop mutation), which were stained with Masson's trichrome, illustrated the massive interstitial fibrosis and myocardial disarrangement (arrows). Scale bar, $50 \mu \mathrm{m}$. 


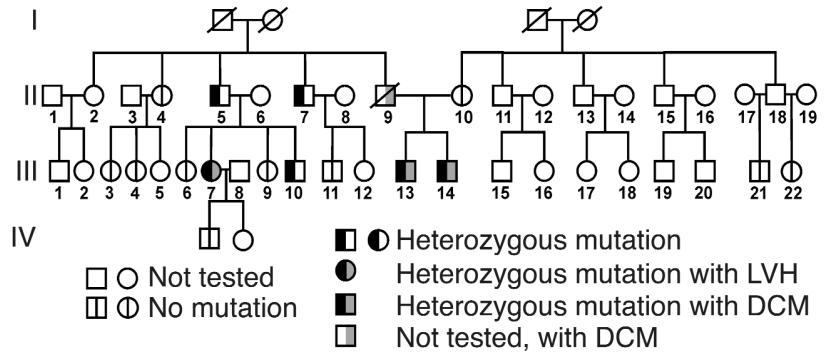

Figure 2

Analysis of inheritance of the T116G mutation in kindred II. Pedigree for the presence or absence of the T116G mutation in four generations. Two brothers (probands III-13 and III-14) who were heterozygous for the L39stop mutation presented with a dilated cardiomyopathy. Their deceased father, II-9, was also diagnosed with dilated cardiomyopathy. Squares represent males and circles represent females. A line denotes that the patient is deceased.

that mutations in this domain can be associated with either an increase in or a loss of the inhibitory effect of PLN on SERCA2a (21).

The extended pedigree of the index case is shown in Figure $1 \mathrm{~b}$. Both homozygous individuals developed severe dilated cardiomyopathy requiring cardiac transplantation. For the male, onset of severe heart failure and cardiac transplantation occurred at age 17 , and he died shortly thereafter because of surgical complications. His homozygous sister exhibited only mild echocardiographic left ventricular hypertrophy at age 20 but developed progressive left ventricular dilation and cardiac failure, requiring cardiac transplantation at the age of 28. Histopathological examination of both explanted hearts revealed marked replacement fibrosis and myofibrillar disarrangement (Figure 1c). History and physical examinations of the other family members failed to elicit symptoms or signs of heart failure.

An additional unrelated heterozygous individual was identified in the cohort of 76 patients (III-13 in Figure 2 ). This man presented with a dilated cardiomyopathy at age 28 , having an ejection fraction of $20 \%$, and his heterozygous brother (III-14 in Figure 2) was also diagnosed with a dilated cardiomyopathy at age 27 with an ejection fraction of $25 \%$. Their father had also died of dilated cardiomyopathy at age 57 , and their mother was homozygous for wild-type PLN. Although DNA was not available for genotyping the father, these results indicate that it is likely that he must have carried at least one copy of the mutant gene (Figure 2). Interestingly, when nine heterozygous subjects from the two pedigrees (I-2, I-3, II-1, II-3, II-4, II-9, and III-5 in Figure 1, and III-7 and III-10 in Figure 2) underwent echocardiographic examination, their left ventricular systolic function (ejection fraction, $64.8 \% \pm 2.8 \%$ ) was normal, but four (I-3, II-1, II-3, III-7) exhibited left ventricular hypertrophy (169, 213,293 , and $313 \mathrm{~g}$ for LVM as compared with $127 \pm 10$ $\mathrm{g}$ for normals, $n=7$ ), indicating incomplete penetrance of the cardiomyopathy phenotype (Figures 1 and 2).
Functional consequences of PLN-L39stop on SR Ca ${ }^{2+}$ transport. The function of PLN-L39stop on SR $\mathrm{Ca}^{2+}$ transport, as compared with wild-type PLN, was examined by coexpression with SERCA2a (1:1 ratio) in HEK-293 cells. Reduction in the apparent affinity of SERCA2a for $\mathrm{Ca}^{2+}\left(\mathrm{K}_{\mathrm{Ca}}\right.$ expressed in pCa units) in microsomes isolated from transfected cells provided a measure of the inhibitory function of PLN (21). Coexpression of human wild-type PLN (PLN-WT) with SERCA2a resulted in the anticipated lower apparent affinity for $\mathrm{Ca}^{2+}$, whereas coexpression of SERCA2a with PLN-L39stop had no effects (Figure 3 , red). Studies were also designed to simulate the heterozygous state, in which wild-type and mutant PLN were coexpressed with SERCA2a. The same decrease in apparent $\mathrm{Ca}^{2+}$ affinity was observed in "heterozygous" cells as in "homozygous wild-type" cells, indicating that the PLN-L39stop mutant does not exert a dominant-negative effect on PLN-WT (Figure 3, green).

Functional consequences of PLN-L39stop on myocyte mechanics and $\mathrm{Ca}^{2+}$ cycling. The effects of PLN-L39stop on myocyte contractility and $\mathrm{Ca}^{2+}$ homeostasis were examined in isolated adult rat myocytes infected with adenoviral vectors containing either wild-type or L39stop PLN cDNAs. Figure 4 ( $a$ and $b$ ) shows representative recordings of cell length and $\mathrm{Ca}^{2+}$ transients in rat cardiomyocytes. As expected, infection with wild-type PLN decreased the extent of cell shortening and the rates of shortening/contraction $(+\mathrm{dL} / \mathrm{dt})$ and relengthening/relaxation $(-\mathrm{dL} / \mathrm{dt})$, with parallel changes in $\mathrm{Ca}^{2+}$ cycling, as compared with control cells infected with an adenovirus expressing GFP (Figure 4c). In contrast, infection with PLN-L39stop adenovirus did not alter myocyte mechanics or $\mathrm{Ca}^{2+}$ cycling (Figure 4, $c$ and $d$ ).

PLN-L39stop expression and localization in HEK-293 cells. Taken together, the above findings indicate that the truncated mutant PLN-L39stop was either inactive or

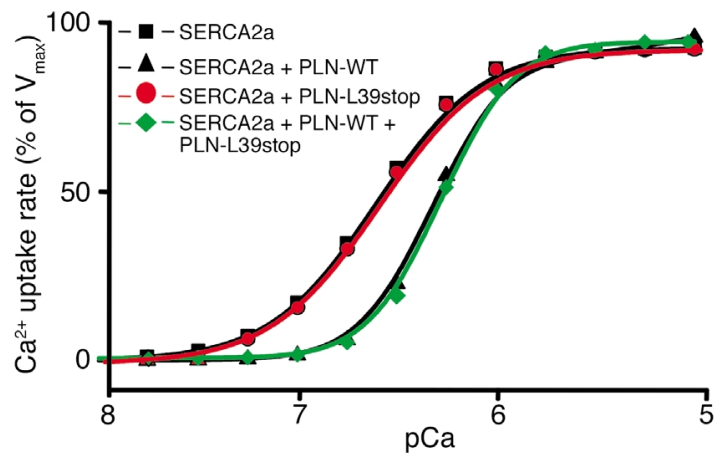

Figure 3

Effect of wild-type and homozygous or heterozygous mutant PLNL39stop on the $\mathrm{Ca}^{2+}$ affinity of SERCA2a. HEK-293 cells were cotransfected with wild-type, homozygous, or heterozygous mutant PLN cDNA and SERCA2a cDNA, and the rates of $\mathrm{Ca}^{2+}$ uptake were measured. The results are representative of two nearly identical and independent experiments. $V_{\max }$, maximum velocity of $\mathrm{Ca}^{2+}$ uptake. 


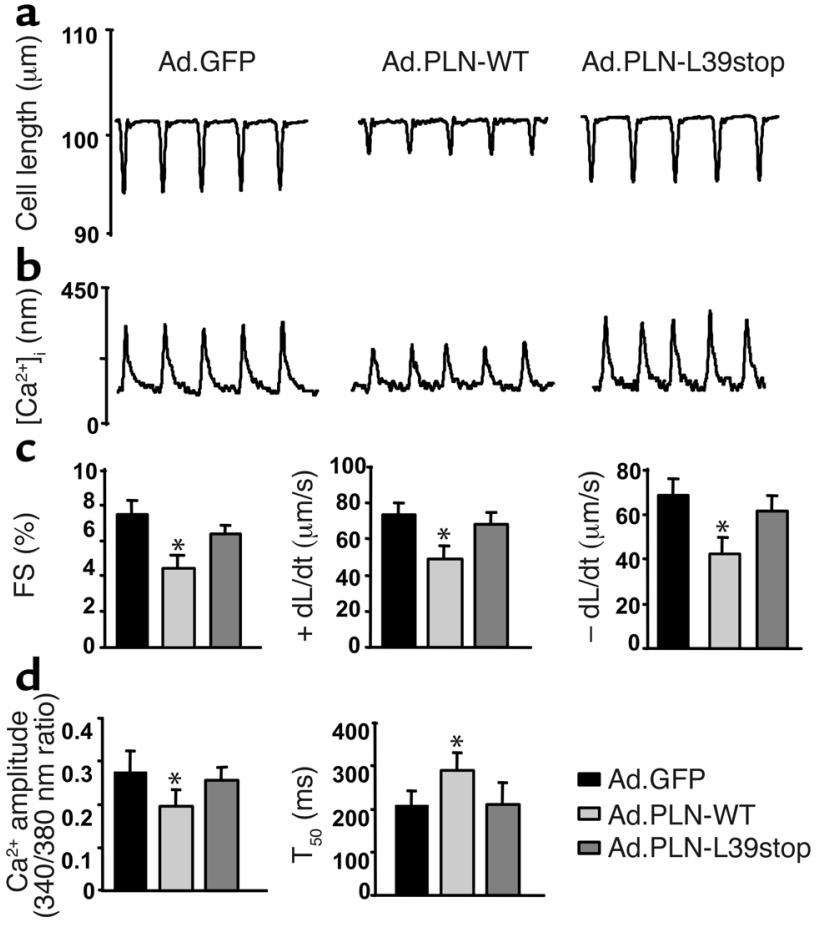

not expressed. Suggesting the latter, the PLN-L39stop protein product could not be detected in Western blots of microsomal fractions from transfected HEK-239 cells (Figure 5a, left panel). However, PLN-L39stop truncated peptide was detected in very small amounts

\section{Figure 4}

Effect of PLN-L39stop mutant on adult rat cardiac myocyte mechanics and $\mathrm{Ca}^{2+}$ kinetics. Shown are representative recordings of cell shortening (a) and the $\mathrm{Ca}^{2+}$ transient (b) in cardiomyocytes isolated from adult rat hearts infected with Ad.GFP, Ad.PLN-WT, and Ad.PLN-L39stop. Myocytes were stimulated at $0.5 \mathrm{~Hz}$ at $25^{\circ} \mathrm{C}$. (c) Percent myocyte fractional shortening (FS\%), rate of contraction $(+\mathrm{dL} / \mathrm{dt})$, and rate of relaxation $(-\mathrm{dL} / \mathrm{dt}) .(\mathbf{d}) \mathrm{Ca}^{2+}$ transient amplitude $\left(340 / 380 \mathrm{~nm}\right.$ ratio) and time to $50 \%$ decay of the $\mathrm{Ca}^{2+}$ signal $\left(\mathrm{T}_{50}\right)$. A minimum of 16-20 cells was studied from each of three individual preparations. Values are means $\pm \mathrm{SEM} ;{ }^{*} P<0.05$.

in the insoluble sedimented fraction from douncehomogenized cells (Figure 5a, right panel). This lower-molecular-weight protein represented monomeric PLN-L39stop, consistent with the previously established fact that PLN oligomerization occurs in the transmembrane domain $(21,26,27)$.

Confocal microscopy in HEK-293 cells transfected with PLN-L39stop revealed detectable immunoreactive protein signals in only $2.3 \%$ of fluorescent cells per coverslip, as compared with PLN-WT transfectants $(23 \pm 5$ vs. $995 \pm 148$ fluorescent cells per coverslip for PLN-L39stop vs. PLN-WT, respectively; $P<0.0002$ ) (Figure $5 b$ ). Where PLN-L39stop protein was detected, its fluorescence was localized primarily to the cell membrane (Figure 5, c and d, lower panels), whereas the abundantly expressed PLN-WT was restricted to the endoplasmic reticulum (Figure 5, c and d, upper panels). a
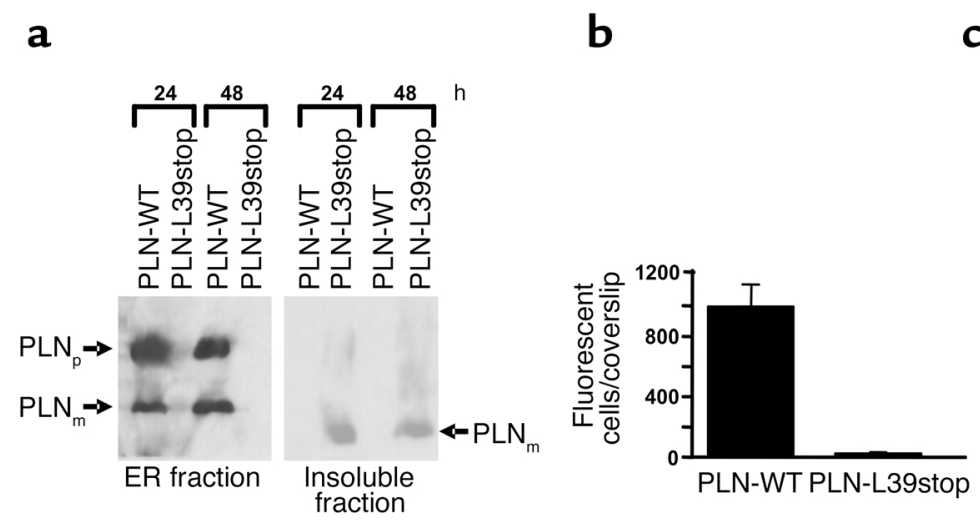

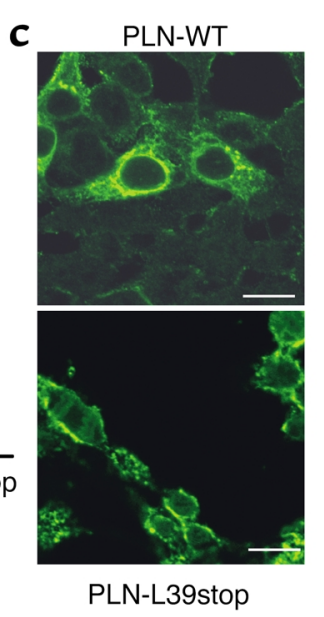

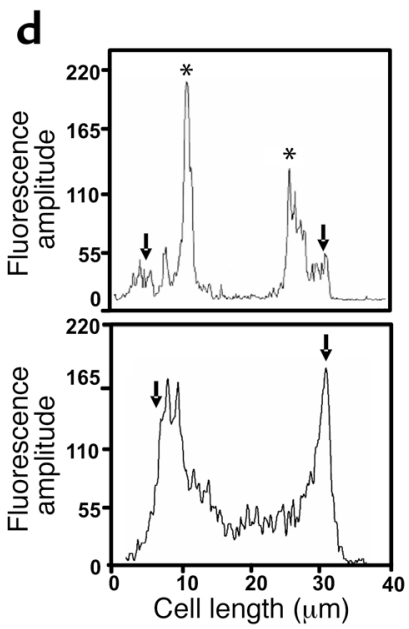

\section{Figure 5}

Expression and localization of PLN-WT and PLN-L39stop mutant in HEK-293 cells. (a) Immunoblot analyses of endoplasmic reticulum microsomes (left panel) and insoluble fractions (right panel) obtained at 24 and 48 hours after transfections. Note the absence of PLN-L39stop in the endoplasmic reticulum fraction, although detectable protein levels are found in the insoluble fraction. PLN ${ }_{p}, P_{L} N$ pentamer; PLN ${ }_{m}, P_{L N}$ monomer. (b) Quantitation of the number of fluorescent cells on PLN-WT and PLN-L39stop transfected coverslips. Cultures were transfected with equal amounts of plasmid DNA, and 48 hours later the number of fluorescent cells was counted on the entire coverslip. (c) Immunofluorescence of PLN-WT and PLN-L39stop transfected cells analyzed by confocal microscopy 48 hours after transfection. In PLN-WT transfected cells, immunofluorescence is exclusively in the endoplasmic reticulum, whereas a clearly distinct and plasma membrane-associated staining pattern is observed in the PLN-L39stop transfected cells. Scale bar, $30 \mu \mathrm{m}$. (d) Fluorescence intensity was quantitated using available Leica software. For these assays, a straight profile line was drawn across the center of the cell and fluorescence amplitude was plotted. Arrows mark the edge of the cell and asterisks mark the ER. In PLN-WT transfected cells (upper panel), immunofluorescence is found within the interior of the cell, whereas in PLN-L39stop transfected cells (lower panel), staining is found enriched at the outer edge of the cell. 

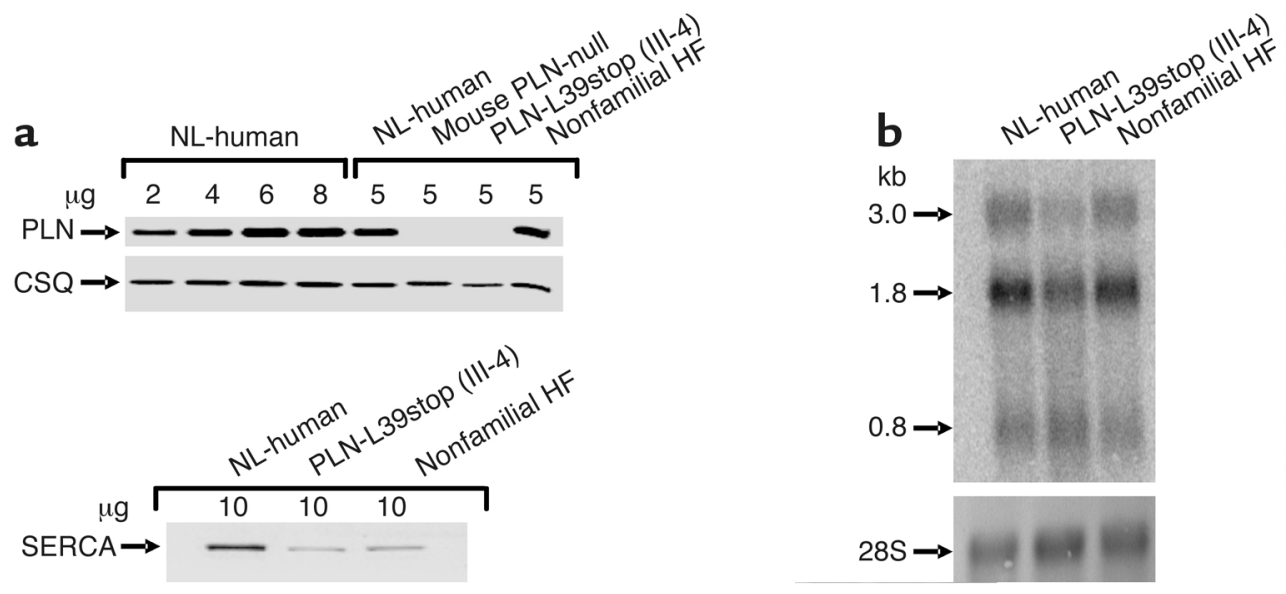

\begin{abstract}
Figure 6
SR proteins and PLN expression levels in explanted heart tissue of proband III-4 in kindred I. (a) Quantitative immunoblotting of PLN, SERCA2a, and calsequestrin in explanted heart tissue of homozygous proband III-4, a nonfamilial heart failure patient, and a normal donor subject as well as the PLN-null mouse. Proteins were visualized with specific antibodies against PLN, SERCA2, and calsequestrin and quantified against a standard obtained from normal human donor heart. (b) Autoradiographic images of oligonucleotide-probed Northern blot of PLN (upper panel), using cardiac RNA from normal individuals and those with PLN-L39stop (III-4) and nonfamilial heart failure. The radiolabeled 60-base oligonucleotide, antisense to the PLN coding region, recognizes the three major PLN mRNAs (0.8, 1.8, and 3.0 kb). These blots were stained with methylene blue to demonstrate the RNA integrity and loading (lower panel). CSQ, calsequestrin; HF, heart failure patient; NL, normal donor.
\end{abstract}

To confirm these findings in human hearts, immunoblot analysis of ventricular myocardium from normal humans, the PLN gene knockout mouse, the explanted heart of PLN-L39stop homozygous patient III-4, and a patient with nonfamilial idiopathic dilated cardiomyopathy was performed. As shown in Figure 6a, the PLN-L39stop homozygous ventricle had no detectable PLN immunoreactivity. As expected, calsequestrin expression was not altered (Figure 6a), and SERCA2a expression was decreased (by about 50\%) in both the PLN-L39stop patients and the patients with idiopathic cardiomyopathy (28). Consistent with the view that the expression defect was at the level of PLN translation or protein turnover, PLN mRNA was readily detectable in PLNL39stop homozygous myocardium, albeit at lower levels than in normal hearts or hearts with idiopathic cardiomyopathy (Figure 6b, upper panel).

\section{Discussion}

We describe here individuals with dilated cardiomyopathy who possess a truncation mutation in the PLN gene that results in an absence of detectable PLN protein - that is, PLN null. This experiment of nature is therefore analogous to previously published PLN gene ablation or "knockout" studies in mice (11, 12). The most important finding is that PLN ablation in humans appears to have effects that are the opposite of those observed with PLN ablation in mice. Whereas the PLN-null mouse displays chronically increased basal cardiac contractile function without developing heart failure, even in advanced age (13), both human subjects homozygous for the
PLN-L39stop mutation developed severe dilated cardiomyopathies requiring cardiac transplantation at an early age, and two heterozygous individuals from an independent family also developed delayed dilated cardiomyopathy. Although our findings indicate the virtual absence of PLN in the homozygous hearts, we cannot rule out the possibility that a highly unstable/rapidly degraded and inactive form of PLN, associated with the PLN-L39stop mutation, may initiate human dilated cardiomyopathy.

The mouse has been used extensively as a model system for human disease and for the design of appropriate therapies, but the phenotype of mouse models may differ from the phenotypes observed in human diseases with the corresponding genotype $(29,30)$. In this study, the surprising difference between human and mouse PLN-null cardiac phenotypes emphasizes the need to better understand inherent differences in cardiac physiology and $\mathrm{Ca}^{2+}$-cycling mechanisms between mice and humans (31). The mouse heart beats over 600 times per minute, or about 10 times faster than the human heart, indicating different mechanisms regulating the dynamic balance of cardiomyocyte $\mathrm{Ca}^{2+}$ fluxes. Whereas $\mathrm{Ca}^{2+}$ removal from the cytosol during cardiac relaxation in mice relies almost exclusively on SERCA2a (92\% of total), in humans $\mathrm{Na}^{+} / \mathrm{Ca}^{2+}$ exchanger activity accounts for approximately one third of $\mathrm{Ca}^{2+}$ removal, with SERCA2a function largely responsible for the remainder (32-34). The basic ventricular motor proteins are also different, with $\alpha$-myosin heavy chain predominating in adult mice but $\beta$-myosin heavy chain predominating in humans (30). An absence of PLN in 
mouse hearts, already operating close to their theoretical maximum, might therefore be less deleterious than in the slower-beating human hearts, in part through the regulatory effects of PLN.

In human subjects homozygous for the PLNL39stop mutation, PLN is absent and SERCA2a should be tonically disinhibited - that is, activated - with enhancement of inotropy and lusitropy analogous to that observed in PLN-null mice $(11,12)$. Increased cardiac work over a period of years could conceivably lead to ventricular hypertrophy, as seen in some of the heterozygous PLN-L39stop individuals, which may then progress to ventricular failure as observed in the homozygous mutants. Indeed, there are numerous examples of a direct association between the development of heart failure and chronic inotropic stimulation, such as pheochromocytoma (15) and catecholamine cardiomyopathies (16), as well as the increased incidence of heart failure from genetic polymorphisms that augment norepinephrine release and cardiac $\beta_{1}$-adrenergic receptor ( $\beta A R$ ) function (35). For each of these conditions, prolonged, unregulated stimulation of the human heart causes or accelerates the progression of heart failure, and the same may be true of the PLN-L39stop mutant. On the other hand, chronic suppression of the $\beta$-adrenergic signaling pathway stimulatory effects by a dominant R9C-PLN mutant also results in human cardiomyopathy and heart failure (36). Thus, a fine balance between the degree of SERCA2a inhibition and augmentation by PLN is essential for normal cardiac function.

The variable expression of the clinical phenotype, elicited by PLN-L39stop, is not unusual in inherited cardiomyopathy (17). In this study, heterozygosity of PLN-L39stop was associated with hypertrophy in some members of both families and with overt dilated cardiomyopathy in other members. As such, environmental perturbations are likely to contribute to the mechanism by which absence of functional PLN induces cardiac dysfunction. For example, in PLNnull mice, the persistently enhanced inotropic state impairs functional recovery from ischemia (37), and chronic $\beta$-adrenergic stimulation increases the incidence of ventricular failure after ischemia or pressure overload (38). Likewise, there are undoubtedly as-yetunidentified genetic influences that can modify the PLN-L39stop phenotype, as strongly suggested by the development of dilated cardiomyopathy in two heterozygous subjects from one of our kindreds but ventricular hypertrophy with normal cardiac function in heterozygous individuals from the other kindred. Future studies in additional human kindreds, and in PLN mice with different genetic backgrounds, will help to resolve this issue.

In conclusion, a genetic mutation resulting in lack of expression of PLN protein was identified in individuals from two families with inherited dilated cardiomyopathy. To our knowledge, this is the first instance of a human equivalent of the PLN-null mouse. The apparent pathological effects of absent PLN in human hearts contrast strikingly with benefits observed in many, although not all (39), mouse heart failure models. These data emphasize a general concern that targeted therapies whose design is based exclusively on results of studies in rodent models, in which phenotypes can differ radically from those observed in the corresponding human genetic condition, may not ultimately be successful in human disease. A specific cautionary note is sounded as to whether drugs targeted to inhibition of PLN will ultimately have a therapeutic role in chronic, as compared with acute, human heart failure.

\section{Acknowledgments}

The cooperation of family members and collaborating physicians in the Cardiology Division at the University of Cincinnati and Onassis Cardiac Surgery Center in Athens, Greece, is appreciated. This research was supported by NIH grants HL-26057, HL-64018, and HL-52318 to E.G. Kranias and grants HL-58010, HL52318, and HL-59888 to G.W. Dorn II; by the Heart and Stroke Foundation of Ontario; and by grant MT- 12545 from the Canadian Institutes of Health Research to D.H. MacLennan. A.O. Gramoli$\mathrm{ni}$ is a postdoctoral fellow of the Canadian Institutes of Health Research.

1. Cohn, J.N. 1997. Current concepts in the treatment of congestive heart failure. Cardiology. 88(Suppl. 2):2-6.

2. Ho, K.K., Pinsky, J.L., Kannel, W.B., and Levy, D. 1993. The epidemiology of heart failure: the Framingham Study. J. Am. Coll. Cardiol. 22:6A-13A.

3. Hasenfuss, G. 1998. Alterations of calcium-regulatory proteins in heart failure. Cardiovasc. Res. 37:279-289.

4. Beuckelmann, D.J., Nabauer, M., and Erdmann, E. 1992. Intracellular calcium handling in isolated ventricular myocytes from patients with terminal heart failure. Circulation. 85:1046-1055.

5. Endo, M. 1977. Calcium release from the sarcoplasmic reticulum. Physiol. Rev. 57:71-108.

6. Brittsan, A.G., and Kranias, E.G. 2000. Phospholamban and cardiac contractile function. J. Mol. Cell. Cardiol. 32:2131-2139.

7. Meyer, M., et al. 1995. Alterations of sarcoplasmic reticulum proteins in failing human dilated cardiomyopathy. Circulation. 92:778-784.

8. Haghighi, K., et al. 2001. Superinhibition of sarcoplasmic reticulum function by phospholamban induces cardiac contractile failure. J. Biol. Chem. 276:24145-24152.

9. Schmidt, A.G., et al. 2002. Structural and functional implications of the phospholamban hinge domain: impaired $\mathrm{SR} \mathrm{Ca}^{2+}$ uptake as a primary cause of heart failure. Cardiovasc. Res. 56:248-259.

10. Hoshijima, M., et al. 2002. Chronic suppression of heart-failure progression by a pseudophosphorylated mutant of phospholamban via in vivo cardiac rAAV gene delivery. Nat. Med. 8:864-871.

11. Luo, W., et al. 1994. Targeted ablation of the phospholamban gene is associated with markedly enhanced myocardial contractility and loss of beta-agonist stimulation. Circ. Res. 75:401-409.

12. Hoit, B.D., Khoury, S.F., Kranias, E.G., Ball, N., and Walsh, R.A. 1995. In vivo echocardiographic detection of enhanced left ventricular function in gene-targeted mice with phospholamban deficiency. Circ. Res. 77:632-637.

13. Slack, J.P., et al. 2001. The enhanced contractility of the phospholamban-deficient mouse heart persists with aging. J. Mol. Cell. Cardiol. 33:1031-1040.

14. Desai, K.H., Schauble, E., Luo, W., Kranias, E., and Bernstein, D. 1999. Phospholamban deficiency does not compromise exercise capacity. Am. J. Physiol. 276:H1172-H1177.

15. Cohn, J.N., et al. 1984. Plasma norepinephrine as a guide to prognosis in patients with chronic congestive heart failure. N. Engl. J. Med. 311:819-823.

16. Bristow, M.R., Shakar, S.F., Linseman, J.V., and Lowes, B.D. 2001. Inotropes and beta-blockers: is there a need for new guidelines? J. Card. Fail. 7:8-12. 
17. Franz, W.M., Muller, O.J., and Katus, H.A. 2001. Cardiomyopathies: from genetics to the prospect of treatment. Lancet. 358:1627-1637.

18. McTiernan, C.F., et al. 1999. The human phospholamban gene: structure and expression. J. Mol. Cell. Cardiol. 31:679-692.

19. Levy, D., Garrison, R.J., Savage, D.D., Kannel, W.B., and Castelli, W.P. 1990. Prognostic implications of echocardiographically determined left ventricular mass in the Framingham Heart Study. N. Engl. J. Med. 322:1561-1566.

20. Asahi, M., Kimura, Y., Kurzydlowski, K., Tada, M., and MacLennan, D.H 1999. Transmembrane helix M6 in sarco(endo)plasmic reticulum $\mathrm{Ca}^{2+}$ ATPase forms a functional interaction site with phospholamban. Evidence for physical interactions at other sites. J. Biol. Chem. 274:32855-32862.

21. Kimura, Y., Kurzydlowski, K., Tada, M., and MacLennan, D.H. 1997. Phospholamban inhibitory function is activated by depolymerization. J. Biol. Chem. 272:15061-15064.

22. He, T.C., et al. 1998. A simplified system for generating recombinant adenoviruses. Proc. Natl. Acad. Sci. U. S. A. 95:2509-2514.

23. Zhang, X.Q., et al. 2001. Overexpression of $\mathrm{Na}^{+} / \mathrm{Ca}^{2+}$ exchanger alters contractility and SR $\mathrm{Ca}^{2+}$ content in adult rat myocytes. Am. J. Physiol. Heart Circ. Physiol. 281:H2079-H2088.

24. Grynkiewicz, G., Poenie, M., and Tsien, R.Y. 1985. A new generation of $\mathrm{Ca}^{2+}$ indicators with greatly improved fluorescence properties. J. Biol. Chem. 260:3440-3450.

25. Brittsan, A.G., Carr, A.N., Schmidt, A.G., and Kranias, E.G. 2000. Maximal inhibition of SERCA2 $\mathrm{Ca}^{2+}$ affinity by phospholamban in transgenic hearts overexpressing a non-phosphorylatable form of phospholamban. J. Biol. Chem. 275:12129-12135.

26. Arkin, I.T., et al. 1994. Structural organization of the pentameric transmembrane alpha-helices of phospholamban, a cardiac ion channel. EMBO J. 13:4757-4764.

27. Simmerman, H.K., Kobayashi, Y.M., Autry, J.M., and Jones, L.R. 1996. A leucine zipper stabilizes the pentameric membrane domain of phospholamban and forms a coiled-coil pore structure. J. Biol. Chem. 271:5941-5946.
28. Dash, R., Frank, K.F., Carr, A.N., Moravec, C.S., and Kranias, E.G. 2001 Gender influences on sarcoplasmic reticulum $\mathrm{Ca}^{2+}$-handling in failing human myocardium. J. Mol. Cell. Cardiol. 33:1345-1353.

29. Geisterfer-Lowrance, A.A., et al. 1996. A mouse model of familial hypertrophic cardiomyopathy. Science. 272:731-734.

30. James, J., et al. 2002. Transgenic rabbits expressing mutant essential light chain do not develop hypertrophic cardiomyopathy. J. Mol. Cell. Cardiol. 34:873-882.

31. Kass, D.A., Hare, J.M., and Georgakopoulos, D. 1998. Murine cardiac function: a cautionary tail. Circ. Res. 82:519-522.

32. Bers, D.M. 2002. Cardiac excitation-contraction coupling. Nature. 415:198-205.

33. Li, L., Chu, G., Kranias, E.G., and Bers, D.M. 1998. Cardiac myocyte calcium transport in phospholamban knockout mouse: relaxation and endogenous CaMKII effects. Am. J. Physiol. 274:H1335-H1347.

34. Hove-Madsen, L., and Bers, D.M. 1993. Sarcoplasmic reticulum Ca ${ }^{2+}$ uptake and thapsigargin sensitivity in permeabilized rabbit and rat ventricular myocytes. Circ. Res. 73:820-828.

35. Small, K.M., Wagoner, L.E., Levin, A.M., Kardia, S.L., and Liggett, S.B. 2002 . Synergistic polymorphisms of $\beta 1$ - and $\alpha 2 \mathrm{C}$-adrenergic receptors and the risk of congestive heart failure. N. Engl. J. Med. 347:1135-1142.

36. Schmitt, J.P., et al. 2003. Phospholamban missense mutation Arg9Cys causes dominant dilated cardiomyopathy in man and mouse. Circulation. 106(Suppl. II):31. (Abstr.)

37. Cross, H.R., Kranias, E.G., Murphy, E., and Steenbergen, C. 2003. Ablation of PLB exacerbates ischemic injury to a lesser extent in female than male mice: protective role of NO. Am. J. Physiol. Heart Circ. Physiol. 284:H683-H690.

38. White, D.C., et al. 2000. Preservation of myocardial beta-adrenergic receptor signaling delays the development of heart failure after myocardial infarction. Proc. Natl. Acad. Sci. U. S. A. 97:5428-5433.

39. Song, Q., et al. 2003. Rescue of cardiomyocyte dysfunction by phospholamban ablation does not prevent ventricular failure in genetic hypertrophy. J. Clin. Invest. 111:859-867. doi:10.1172/JCI200316738. 